\title{
Biomass-derived activated carbon as high-performance non- precious electrocatalyst for oxygen reduction
}

Keliang Wang, Hui Wang, Shan Ji, Hanqing Feng, Vladimir Linkov and Rongfang Wang

\begin{abstract}
A new type of Fe and $\mathrm{N}$ doped carbon material is synthesized by pyrolyzing ferric chloride doped egg white (EW) and the proposed synthetic route is easy, green, and low-cost. In addition, the as-prepared sample exhibits a feasible magnetism and comparable oxygen reduction reaction (ORR) activity to commercial Pt/C.
\end{abstract}

As the cathodic ORR plays an important role in the performance of a fuel cell, ${ }^{1,2}$ efficient ORR electrocatalysts are highly desirable for practical applications. So far, Pt and its alloys have long been regarded as the most effective catalysts for the ORR in fuel cells.3,4 However, their large-scale commercial applications have been hindered by high costs. 5 Besides, they still suffer from serious intermediate tolerance, anode crossover, sluggish kinetics, and poor stability in an electrochemical environment. ${ }^{6-8}$ For these sakes, extensive research has been expected to solve the problems that involve (i) replacing the noble metal by alloy to reduce the consumption of novel metals and to lower the cost;9,10 (ii) assuming certain novel carbon materials with particular shapes like carbon nanotubes (CNTs) and graphene to promote the conductivity of the catalyst or the intermediate product trans- fer;,11,12 (iii) the introduction of transition metals (such as $\mathrm{Fe}, \mathrm{Co})^{13,14}$ as well as metal oxides (such as $\mathrm{Fe}_{3} \mathrm{O}_{4}$ and $\mathrm{CoO})^{15,16}$ to boost the ORR; (iv) doping some $\mathrm{N}, \mathrm{B}, \mathrm{P}$, and $\mathrm{S}$ atoms ${ }^{17-20}$ to modify the electrocatalysts to enhance the methanol tolerance. Among these explorations, it is confirmed that nitrogen-doped carbon materials not only exhibit high catalytic activity, long-term stability, and excellent methanol tolerance in alkaline media, but also possess the advantages of low cost and environmental friendliness.

According to the theory of Dai et al., ${ }^{21}$ the high catalytic activity by doping $\mathrm{N}$ may be attributed to the larger electronegativity of $\mathrm{N}$ compared to $\mathrm{C}$ atoms. And, $\mathrm{N}$ atoms can create positive charge density on the adjacent $\mathrm{C}$ atoms, which results in the very favorable adsorption of $\mathrm{O}_{2}$. Consequently, intense research on the ways to modify the carbon materials with nitrogen atom has been greatly inspired. To our knowledge, the source of nitrogen is always derived from expensive organic monomer containing nitrogen element ${ }^{22}$ or $\mathrm{NH}_{3} \cdot{ }^{12}$ However, these 
nitrogen-containing compounds are either expensive or harmful to human health. In addition, it is complicated to prepare the nitrogen-doped and non- precious electrocatalysts. Thus, a simple and convenient method to synthesize a new kind of ORR electrocatalysts with the features of low price, green origin as well as excellent performance can be expected.

Considering that several species of natural biomass were successfully given to be carbon materials and these materials showed potential applications in a variety of fields, particularly in fuel cells, egg white (EW) could be a promising candidate. Since EW abounds with carbon, nitrogen and oxygen elements owing to the existence of proteins, it is expected to provide an alternative precursor to synthesize advantageous nanomaterials. Herein, we demonstrated an easy and green strategy to synthesize a new type of N, Fe doped non-precious electrocatalyst by using EW as the sources of carbon and nitrogen sources. This new type of electrocatalyst (Fe-CEW) not only showed favorable magnetic properties but also exhibited surprising electrocatalytic activity towards ORR. Verified by the electrochemical experiments, the as- prepared samples had ORR catalytic activity comparable to the commercial carbon-supported Pt catalyst and had superior stability, thus leading to a new high performance catalyst for ORR. First of all, the practical composition of CEW and FeCEW were evaluated by both elemental analysis. The analytical results indicate that CEW consists of $\mathrm{N}$ (6.9 wt\%), $\mathrm{C}$ (62.7 wt\%), and the reminder inorganic salt residue like $\mathrm{K}$, Na, Ca etc.; Fe-CEW consists of N (4.4 wt\%) and C (48.4 wt\%). Based on the ratio of each element, metallic Fe is calculated to be 16.8 wt\%. The XRD patterns of CEW and FeCEW (Fig. S1, ESI 3 ) show two peaks located at $2 \mathrm{~h} \# 25 \mathrm{u}$ and $44 \mathrm{u}$, which can be attributed to (002) and (101) diffraction peaks from different forms of carbon, referred to turbostratic carbon (carbon black) and grapheme carbon (graphi- tic structure), 23 respectively. The other diffraction peaks at ca. 28u, 31u, 41u, 45u in the two curves are assigned to certain inorganic salt residue of $\mathrm{K}, \mathrm{Na}$, Ca etc. originated from $\mathrm{CEW}$. Besides, a diffraction peak at $2 \mathrm{~h}=35 \mathrm{u}$ can be observed for Fe-CEW sample, which is associated with the iron/carbon-black composite nanoparticle, ${ }^{24}$ manifesting that the doped $\mathrm{Fe}$ is bonded with EW after heat treatment. In the Raman spectrum of XC-72, CEW and FeCEW in Fig. S2, ESI,3 the peaks at $\mathrm{y} 1310$ and $\mathrm{y} 1590 \mathrm{~cm}^{21}$ assigned to the $\mathrm{D}$ and $\mathrm{G}$ band, respectively, can be observed. The peak intensity ratio of the $\mathrm{D}$ to $\mathrm{G}$ band (ID/IG) for the $\mathrm{XC}-72$ and $\mathrm{CEW}$ is 1.03 and 1.14, respectively, suggesting that the CEW shows more defect sites (edge plans) and poorer electric conductivity than the XC-72 does. ${ }^{25}$ In terms of the raman spectrum of CEW and Fe-CEW, both the D band and G band do not significantly change in ether width or shape apart from the $I_{\mathrm{D}} / I_{\mathrm{G}}$. The $I_{\mathrm{D}} / I_{\mathrm{G}}$ ratio of Fe-CEW is 1.07, closed to the value of XC-72 but smaller than that of CEW (1.14), indicating that (i) the Fe-CEW exhibits less defects sites than the CEW, which may be caused from the original defect sites that is covered by the doped Fe atoms, as reported in literature for the 1-aminopyrene-functionalized multi- walled carbon nanotubes; 25 (ii) the Fe-CEW possesses much better electric conductivity compared to CEW, which means a higher current density and excellent performance for ORR. ${ }^{26}$ Fig. $\mathrm{S}_{3}, \mathrm{ESI}_{3}$ shows the surface functional groups of the carbon nitride materials are 
characterized by FT-IR. Fig. S4, ESI3 displays TGA and DTG profiles of the decomposition process of EW and Fe doped egg white (Fe-EW), from which we can obtain information on residual masses at different temperatures. As shown in Fig. S4a, ESI, 3 two stages are included in the pyrolysis process. A slight weight loss occurs during the first stage ranging from the starting temperature to y2oo $\mathrm{uC}$, which can be related to the elimination of water (dehydration). The second stage, from y20o uC to the end, is characterized by a major loss, which corresponds to the main pyrolysis process (devolatilization). It proceeds with a high rate in the initial period but keep a comparatively steady rate in the later stage, demonstrating that prior to forming the nitrogen- doped carbon materials, the carbohydrate in EW is pyrolyzed firstly. With respect to the decomposition process of Fe-EW (as shown in Fig. S4b, ESI3), it can be also divided into two stages: (i) dehydration (from room temperature to y200 $\mathrm{uC}$ ) due to the removal of adsorbed and bound water; (ii) devolatilization under the participation of $\mathrm{FeCl}_{3}$. However, we notice that the second stage in curve $\mathrm{b}$ is absolutely distinct from that in curve $a$. The curve $b$ displays a broader platform region than curve a. We infer that during the pyrolysis process of $\mathrm{EW}$, the reaction of forming the composite of $\mathrm{Fe}-\mathrm{N} / \mathrm{C}$ occurs simultaneously.

Fig. 1 displays the $\mathrm{N}$ 1s spectra for CEW, Fe-CEW and Fe $2 p$ spectra for Fe-CEW. As reported in the literature, ${ }^{12}$ the $\mathrm{N}$ 1s spectrum can be further deconvoluted into three different signals with binding energies of 398.0, 400.0, and $401.3 \mathrm{eV}$, each of which correspond to pyridinic-N, pyrrolic-N and graphitic-N, respectively. Of these three types of $\mathrm{N}$ species, pyridinic- $\mathrm{N}$ and pyrrolic-N, particularly for pyrrolic- $\mathrm{N}$, have a lone electron pair in the plane of the carbon matrix, resulting in an increase of electrondonor property for the catalysts. ${ }^{21}$ The $\mathrm{O}-\mathrm{O}$ bond would be weaken by the bonding between oxygen and nitrogen (and/or the adjacent carbon atom), and thus the reduction of oxygen is facilitated. ${ }^{27}$ The Fe $2 p$ spectrum for Fe-CEW shows two peaks at 726.0 and $711.5 \mathrm{eV}$ (Fig. 1c), corresponding to $\mathrm{Fe} 2 \mathrm{p}_{1} / 2$ and $\mathrm{Fe} 2 \mathrm{p}_{3} / 2$, respectively. The value is similar to that of a certain kind of $\mathrm{Fe}-\mathrm{N} / \mathrm{C}$ compound reported previously. ${ }^{28}$ It could be inferred that $\mathrm{Fe}$ atoms bond with nitrogen and/or carbon. Considering the introduction of $\mathrm{Fe}$, the as-prepared samples may exhibit the properties of magnetism after heat treatment. As shown in Fig. 2a and b, a reasonable amount of samples is dispersed into a standard bottle containing ethanol, and then a magnet is kept closed to the bottle. After a few times, the sample of Fe-CEW is separated from the ethanol solution, revealing that the Fe-CEW sample exhibits good magnetic features and re-disperse properties. This suggests a potential application for targeting and separation. 


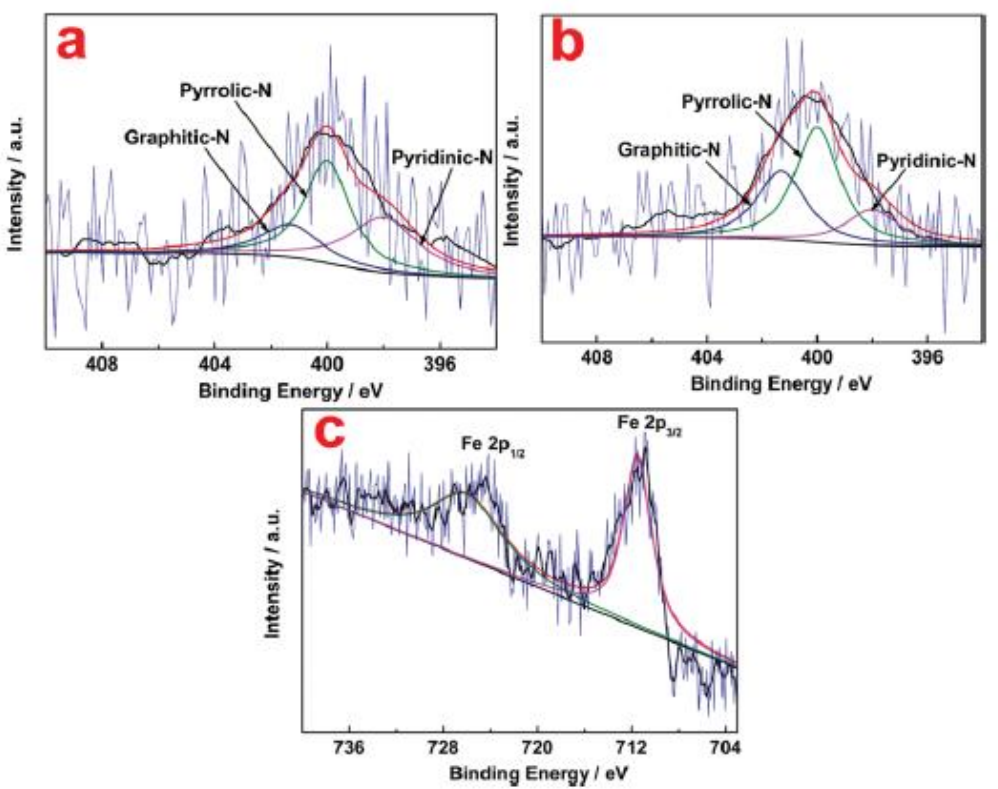

Fig. $1 \mathrm{~N}$ 1s peaks in the XPS spectrum for (a) CEW, (b) Fe-CEW and Fe 2p peaks in the XPS spectrum for (c) Fe-CEW.

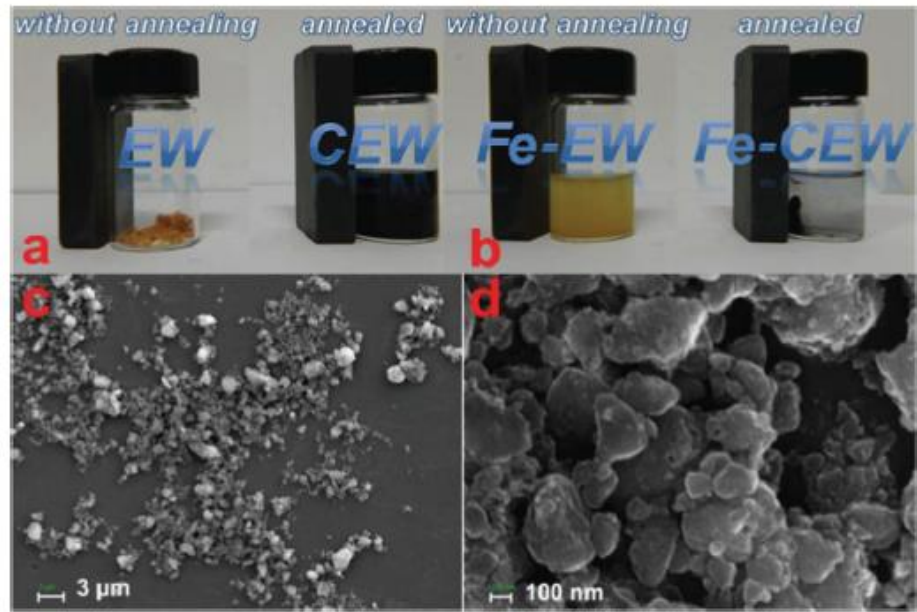

Fig. 2 The separation process of the as-prepared (a) EW, CEW and (b) Fe-EW, FeCEW with magnet; SEM images with different magnification for the as-prepared ( $c$, d) Fe-CEW. 

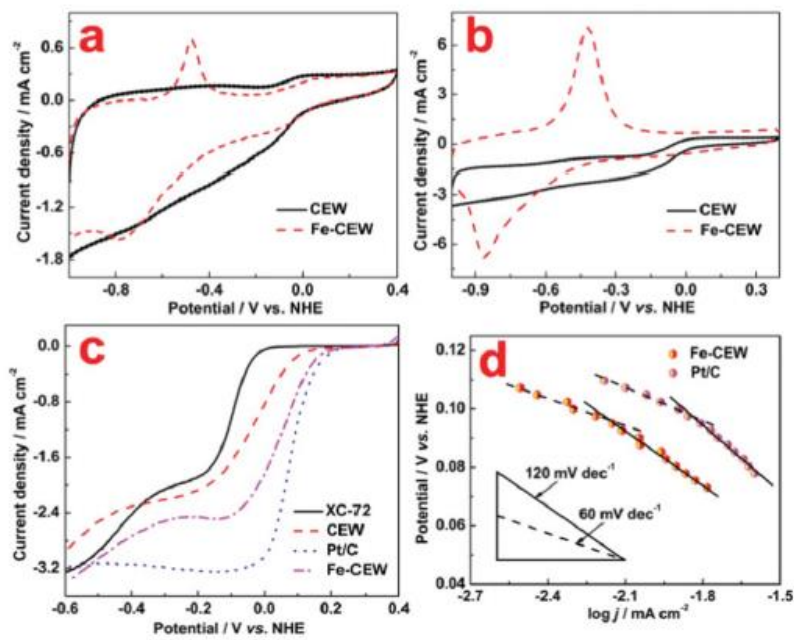

Fig. 3 CV curves for ORR on CEW and Fe-CEW electrocatalysts in $\mathrm{N}_{2}$-sarurated (a) and $\mathrm{O}_{2}$-saturated (b) $0.1 \mathrm{~mol} \mathrm{~L}^{-1} \mathrm{KOH}$ solution; (c) LSV curves for ORR on the XC-72, CEW, Pt/C and Fe-CEW electrocatalysts in $\mathrm{O}_{2}$-saturated $0.1 \mathrm{~mol} \mathrm{~L}^{-1} \mathrm{KOH}$; (d) Tafel plots for ORR on Fe-CEW and Pt/C. Rotation rates: $1600 \mathrm{rpm}$, scan rate: $50 \mathrm{mV} \mathrm{s}^{-1}$ for $\mathrm{CV}$ and $5 \mathrm{mV} \mathrm{s}^{-1}$ for LSV, temperature: $30^{\circ} \mathrm{C}$
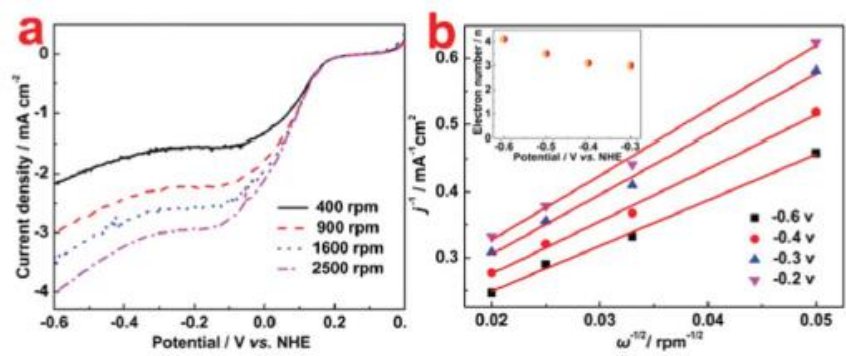

Fig. 4 (a) Current-potential curves for ORR in $\mathrm{O}_{2}$-saturated $0.1 \mathrm{~mol} \mathrm{~L}^{-1} \mathrm{KOH}$ on the Fe-CEW electrode at various rotation rates; (b) Koutecky-Levich $(K-L)$ plots for ORR on the Fe-CEW electrode (data derived from (a)); inset of (b) is the dependence of $n$ on potential for the Fe-CEW electrode.

Fig. 2c and d show scanning electron microscopy (SEM) images of the as- prepared Fe-CEW. It can be observed that Fe-CEW has formed coral-like structure with some anomalous pores, which possibly provides large specific surface area for the interface reaction, stores liquid electrolyte, and promotes the mass transfer and separation.

Cyclic voltammogram (CV) curves on the CEW and Fe-CEW electrodes in $\mathrm{N}_{2}$-saturated and $\mathrm{O}_{2}$-saturated $0.1 \mathrm{~mol} \mathrm{~L}^{21} \mathrm{KOH}$ solutions are shown in Fig. 3a and b. As can be seen, an oxidation peak appears at around $20.47 \mathrm{~V}$ and $20.42 \mathrm{~V}$ in the CV curve of FeCEW in $\mathrm{N}_{2}$ and $\mathrm{O}_{2}$ atmosphere, arising from the $\mathrm{Fe}(\mathrm{II})$ is oxidized to $\mathrm{Fe}(\mathrm{III}),{ }^{24}$ whereas no peaks have response to the curve of CEW. Beyond that, it can also be found that the CV of the Fe- CEW shows a current density comparable with that of CEW in $\mathrm{N}_{2}$ atmosphere, but a larger electric double layer than that of CEW in $\mathrm{O}_{2}$ atmosphere. This result implies that the doped $\mathrm{Fe}$ is involve into the electrochemical process, including the ORR and provides a larger electrochemically accessible area, an area where the 
electrolyte can reach. ${ }^{29}$ To further confirm whether the $\mathrm{N}$ and Fe work in the process of ORR, we conduct the linear sweep voltammograms (LSV) experiments as shown in Fig. 3c. First of all, the LSV curve of CEW is compared with that of XC-72 to verify the function of $\mathrm{N}$ contained in EW. As illustrated in the LSV curves, the half-wave potential of the CEW is $2212 \mathrm{mV}$, which is a shift of $56 \mathrm{mV}$ more positive to that of the XC-72 (2268 $\mathrm{mV})$. Subsequently, the similar method is used to confirm the role of doped Fe. It is found that, once the $\mathrm{Fe}$ is introduced, the half-wave potential of Fe-CEW (2130 mV) shifted positively to around $82 \mathrm{mV}$ compared to $\mathrm{CEW}$, but a little lower $(30 \mathrm{mV})$ than that of $\mathrm{Pt} / \mathrm{C}(2100 \mathrm{mV})$. In terms of the onset potential, it decreases in the order of $\mathrm{Pt} / \mathrm{C}$ and Fe-CEW $(220 \mathrm{mV})>\mathrm{CEW}(181 \mathrm{mV})>\mathrm{XC}-72(40 \mathrm{mV})$ (as shown in Fig. S5, ESI 3 ). Hence, based on the above facts, it has clearly demonstrated a significant enhancement of electro-catalytic activity for Fe-CEW towards the ORR as a result of the introduction of $\mathrm{N}$ and $\mathrm{Fe}$ in respect to CEW and XC-72. Subsequently, to investigate the ORR kinetic character of Fe- CEW and Pt/C, Tafel plots (Fig. 3d) are carried out. In Tafel plots, two well-defined linear regions similar with Tafel slope values are displayed at high and low potentials, respectively. The Tafel slope values of $60 \mathrm{mV} \mathrm{dec} 21$ at high potentials suggest that ORR rate may be determined by migration of adsorbed oxygen intermediates. ${ }^{30}-32$ While at low potentials, the Tafel slope of $120 \mathrm{mV} \mathrm{dec}{ }^{21}$ could be ascribed to the transfer of the first electron as a rate-determining step.31,32 The above results suggest that the ORR mechanism of Fe-CEW may be as similar as that of Pt/C.

Then, rotating disk electrode (RDE) current-potential curves of Fe-CEW at various rotating speeds are plotted in Fig. 4a. The transferred electron numbers per $\mathrm{O}_{2}$ involved in the oxygen reduction at the Fe-CEW electrode is determined by the Koutecky- Levich (K-L) equation as given below

$$
\frac{1}{j}=\frac{1}{j_{k}}+\frac{1}{B \omega^{0.5}}
$$

where $j$ is themeasured currentdensity, $j \mathrm{k}$ is thekinetic current density, $v$ is therotation speed, $B$ is the slope of K-Lplots which can be obtained from Fig. 4 b based on the Levich equation as follows:33,34

$$
B=0.2 n \mathrm{~F}\left(D_{\mathrm{O}_{2}}\right)^{2 / 3} v^{-1 / 6} C_{\mathrm{O}_{2}}
$$

where $\mathrm{n}$ is the number of electrons transferred in the reduction of one $\mathrm{O}_{2}$ molecule in ORR, $\mathrm{F}$ is the Faraday constant $\left(\mathrm{F}=96485 \mathrm{Cmol}^{21}\right), \mathrm{DO}_{2}$ is the diffusion coefficient of $\mathrm{O}_{2}$ in 0.1 $\mathrm{mol} \mathrm{L}^{21} \mathrm{KOH}\left(\mathrm{DO}=1.9610^{25} \mathrm{~cm}^{2} \mathrm{~s}^{21}\right), \mathrm{CO}_{2}$ is the bulk concentration of $\mathrm{O}_{2}$ in the electrolyte $\left(\mathrm{CO}_{2}=1.2610^{26} \mathrm{~mol} \mathrm{~cm}{ }^{23}\right)$, and $\mathrm{n}$ is the kinematic viscosity of the electrolyte $\left(\mathrm{n}=0.01 \mathrm{~cm}^{2}\right.$ $\mathrm{s}^{21}$ ).35 The constant 0.2 is adopted when the rotation speed is expressed in rpm. Calculated

\section{http://repository.uwc.ac.za}


from the slope of the $\mathrm{K}-\mathrm{L}$ plots, the number of the average number of the electrons transferred per $\mathrm{O}_{2}$ molecule for the sample of $\mathrm{Fe}$ - CEW in the process of ORR is 3.4 at a potential ranging from 20.6 to $20.3 \mathrm{~V}$ for Fe-CEW. Typically, the above results demonstrate that the process of ORR on Fe-CEW proceeds close to $4 \mathrm{e}^{2}$ pathway.
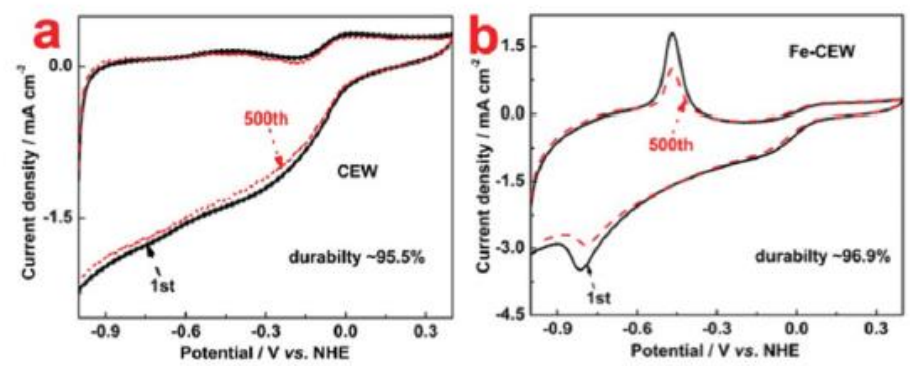

Fig. 5 CVs for ORR at (a) CEW and (b) Fe-CEW electrodes before and after a continuous potentiodynamic swept for 500 cycles in $0.1 \mathrm{~mol} \mathrm{~L}^{-1} \mathrm{KOH}$ under air atmosphere. Rotation rate: $1600 \mathrm{rpm}$, scan rate: $50 \mathrm{mV} \mathrm{s}^{-1}$ for CV, temperature: 30 ${ }^{\circ} \mathrm{C}$.

Finally, the stability of CEW and Fe-CEW is evaluated by CV method. As indicated in Fig. 5a and b, 500 cycles have been measured so that the catalysts can trend to be a steady state, and then the following 500 cycles can be used to assess the stability of the obtained Fe-CEW catalyst. According to the method that reported in literature, 15 the CV shows a reliable stability of Fe-CEW less than $3.1 \%$ current density loss at $0.6 \mathrm{~V}$, in contrast, the deterioration of the CEW catalyst reaches $4.5 \%$. Based on the above result, the Fe-CEW catalyst exhibits a superior durability in alkaline medium, which may be explained as the reported principle 32 that the relative strong strength of covalent $\mathrm{N}-\mathrm{C}$ bond and coordination linkage between $\mathrm{Fe}$ and $\mathrm{N}$.

In summary, a novel approach to prepare Fe and/or $\mathrm{N}$ doped carbon materials is developed by pyrolyzing EWandFe-EW, which can be used as non-precious electrocatalysts for ORR. The synthetic route is green and low-cost, thus providing an original perspective for the development of new catalytic carbon materials in fuel cells. Interestingly, the Fe-CEW exhibits fine magnetism, which suggests a potential application for targeting and separa- tion. Among the as-prepared samples, Fe-CEW exhibits excellent performance for the ORR. It is proved that $\mathrm{N}$ (including pyridinic- $\mathrm{N}$ and pyrrolic-N) and $\mathrm{Fe}$ immensely promote the ORR. In addition, compared to commercial Pt/C, Fe-CEW displays a comparable ORR activity, which suggests that it could be a promising candidate for ORR catalysts in thefuture.

\section{Acknowledgements}

The authors would like to thank the National Natural Science Foundation of China (21163018) and the National Science Foundation for Post-doctoral Scientists of China (20110490847, 2012T50554) for financially supporting this work. 


\section{Notesandreferences}

1. M.-H.Shao, K.SasakiandR.R.Adzic,J.Am.Chem.Soc., 2006, 128, 3526-3527.

2. C. Wang, H. Daimon, T. Onodera, T. Koda and S. Sun, Angew. Chem., Int. Ed., 2008, 47, 35883591.

3. R. Wang, H. Li, S. Ji, H. Wang and Z. Lei, Electrochim. Acta, 2010, 55, 1519-1522.

4. W. Wang, R. Wang, S. Ji, H. Feng, H. Wang and Z. Lei, J. Power Sources, 2010, 195, 3498-3503.

5. T. Iwazaki, H. Yang, R. Obinata, W. Sugimoto and Y. Takasu, J. Power Sources, 2010, 195, 58405847 .

6. Z.Pengand H.Yang, J.Am. Chem.Soc., 2009, 131,7542-7543.

7. R. Bashyam and P.Zelenay, Nature, 2006, 443, 63-66.

8. D.WangandS.Lu,Chem. Commun., 2010, 46,2058-2060.

9. Q. Huang, H.Yang, Y. Tang, T. Lu and D. L. Akins, Electrochem. Commun., 2006, 8, 1220-1224.

10. A.Sarkar,A.V. MuruganandA. Manthiram, J.Mater.Chem., 2009, 19, 159-165.

11. Z.Chen, D. Higgins andZ. Chen, Carbon, 2010, 48, 3057-3065. 12

12. L. Qu, Y. Liu, J.-B. Baek and L. Dai, ACS Nano, 2010, 4, 1321-1326.

13. D. Deng, L. Yu, X. Chen, G. Wang, L. Jin, X. Pan, J. Deng, G. Sun and X. Bao, Angew. Chem., Int. Ed., 2013, 52, 371-375.

14. S. N. Goubert-Renaudin and X. Zhu, J. Electrochem. Soc., 2012, 159, B426-B429.

15. Z.-S. Wu, S. Yang, Y. Sun, K. Parvez, X. Feng and K. Müllen, J. Am. Chem. Soc., 2012, 134, 90829085 .

16. S. Guo, S. Zhang, L. Wu and S. Sun, Angew. Chem., Int. Ed., 2012, 51,11770-11773.

17. W.Yang, T.-P.Fellinger and M.Antonietti, J. Am. Chem. Soc., 2010, 133, 206-209.

18. L. Yang, S. Jiang, Y. Zhao, L. Zhu, S. Chen, X. Wang, Q. Wu, J. Ma, Y. Ma and Z. Hu, Angew. Chem., Int. Ed., 2011, 50, 7132-7135.

19. Z. W. Liu, F. Peng, H. J. Wang, H. Yu, W. X. Zheng and J. Yang, Angew. Chem., Int. Ed., 2011, 50, 3257-3261.

20.Z. Yang, Z. Yao, G. Li, G. Fang, H. Nie, Z. Liu, X. Zhou, X. Chen and S. Huang, ACS Nano, 2011, 6, 205-211.

21. K. Gong, F. Du, Z. Xia, M. Durstock and L. Dai, Science, 2009, 323, 760-764.

22. Y. Li, T. Li, M. Yao and S. Liu, J. Mater. Chem., 2012, 22, 10911-17.

23. G. Wu, Z. Chen, K. Artyushkova, F. H. Garzon and P.Zelenay, ECS Trans., 2008, 16, 159-170.

24. C.-Y. Kao and K.-S. Chou, J. Power Sources, 2010, 195, 2399-2404.

25. Y.L. Hsin, K.C.Hwang andC.-T.Yeh,J.Am.Chem.Soc., 2007, 129, 9999-10010.

26. S. Wang, X. Wang and S. P. Jiang, Langmuir, 2008, 24, 10505-10512.

27. G.Liu,X.Li,P.GanesanandB.N.Popov,Appl.Catal., B,2009, 93,156-165.

28. F. Charreteur, F. Jaouen, S. Ruggeri and J.-P. Dodelet, Electrochim. Acta, 2008, 53, 2925-2938.

29. G. Wu, D. Li, C. Dai, D. Wang and N. Li, Langmuir, 2008, 24, 3566-3575.

30. G.Wu, K. L.More,C.M.JohnstonandP.Zelenay,Science, 2011, 332, 443-447.

31. G. Wu, M. Nelson, S. Ma, H. Meng, G. Cui and P. K. Shen, Carbon, 2011, 49, 3972-3982.

32. H.Xiao,Z.-G. Shao, G.Zhang, Y.Gao, W. Lu and B. Yi, Carbon, 2013, 57, 443-51.

33. D. Yu, Q. Zhang and L. Dai, J. Am. Chem. Soc., 2010, 132, 15127-15129.

34. S. Jiang, C. Zhu and S. Dong, J. Mater. Chem. A, 2013, 1, 3593-3599.

35. K. Tammeveski, K. Kontturi, R. J. Nichols, R. J. Potter and D. J. Schiffrin, J. Electroanal. Chem., 2001, $515,101-112$. 